\title{
Anterolateral lesions of the medial forebrain bundle increase the frequency threshold for self-stimulation of the lateral hypothalamus and ventral tegmental area in the rat
}

\author{
BEVERLEY MURRAY and PETER SHIZGAL \\ Concordia University, Montreal, Quebec, Canada
}

\begin{abstract}
Psychophysical data suggest that descending reward fibers directly link the lateral hypothalamus (LH) and ventral tegmental area (VTA) (Bielajew \& Shizgal, 1982, 1986). As a step toward identifying the nuclei from which these fibers arise, electrolytic lesions were made in the anterior medial forebrain bundle (MFB) and the effect on self-stimulation of both the LH and VTA was examined. Changes in the rewarding effect were inferred from lateral displacements of curves relating the rate of responding to the frequency of the stimulation. Seven rats with lesioning electrodes in the anterior $\mathrm{LH}$ and self-stimulation electrodes in a more caudal portion of the $\mathrm{LH}$ and/or in the anterior VTA served as subjects. Lesions in 5 of the 7 rats displaced the rate-frequency functions for the LH and/or VTA sites toward higher frequencies, a result consistent with a reduction in the rewarding impact of the stimulation. Both of the ineffective lesions were centered in Compartment $\mathrm{C}$ of the anterior MFB, whereas the five effective lesions invaded the more lateral Compartments A, D, and E (Nieuwenhuys, Geeraedts, \& Veening, 1982). These data suggest that projections arising in, coursing through, or terminating in the anterolateral MFB play a role in the rewarding effect of stimulating the LH and the VTA.
\end{abstract}

Since the discovery of intracranial self-stimulation (Olds \& Milner, 1954), researchers have sought to identify the neurons responsible for the rewarding effect. One approach to this challenge (Deutsch, 1964; Gallistel, 1975; Gallistel, Shizgal, \& Yeomans, 1981) entails psychophysical inference of physiological and anatomical characteristics of reward-related cells so that these cells can be distinguished from other neurons concurrently activated by the stimulation. Application of the psychophysical approach has yielded an increasingly detailed portrait of the substrate for self-stimulation of the medial forebrain bundle (MFB) and other structures as well (see Shizgal and Murray, 1989, for a recent review).

The psychophysically derived portrait of the MFB substrate includes fibers that link the lateral hypothalamus (LH) and ventral tegmental area (VTA) (Bielajew \& Shizgal, 1982; Shizgal, Bielajew, Corbett, Skelton, \& Yeo-

\footnotetext{
This work was supported by an operating grant to Peter Shizgal from the Medical Research Council of Canada (MA-8037), a team grant from the Fonds FCAR du Québec (ER-124, P. Shizgal, P.I.), and an infrastructural grant from the Fonds FCAR du Québec (CE-103) to the Centre for Studies in Behavioural Neurobiology (R. A. Wise, P.I.). The authors are grateful to Sandra Boye, Kent Conover, Margaret Forgie, and Meg Waraczynski for their very helpful comments on the manuscript. Correspondence should be addressed to P. Shizgal, Centre for Studies in Behavioural Neurobiology, Concordia University, 1455 de Maisonneuve Blvd. W., Montreal, Quebec, H3G 1M8, Canada. (Tel: 514-848-2191).
}

mans, 1980); at least some portion of the rewarding effect is attributed to the propagation of action potentials in the rostrocaudal direction (Bielajew \& Shizgal, 1986). Estimates of the refractory periods (Yeomans, 1975, 1979) and conduction velocities (Bielajew \& Shizgal, 1982) of these fibers suggest that they are thin and myelinated.

Recordings of single-unit action potentials show that cells in several septal and basal forebrain nuclei give rise to descending MFB fibers with refractory periods, conduction velocities, and trajectories consistent with psychophysically derived estimates (Rolls, 1974; Rompré \& Shizgal, 1986; Shizgal, Schindler, \& Rompré, 1989). This could mean that the neurons in question are components of the directly stimulated substrate or that these neurons subserve some other function but happen to bear a close resemblance to the cells responsible for the rewarding effect. Lesion experiments offer a means of distinguishing between these interpretations. If a given nucleus contains somata that give rise to fibers composing part of the directly stimulated substrate for self-stimulation of the MFB, then damage to that nucleus ought to weaken the rewarding impact of MFB stimulation.

Early efforts to identify the substrate for brain stimulation reward (BSR) often involved brain lesions. Unfortunately, the rate of operant responding for a fixed set of stimulation parameters served as the measure of the rewarding effect in most of the early experiments (e.g., Boyd \& Gardner, 1967; Lorens, 1966; Olds \& Olds, 1969). This method has been shown to confound changes 
in reward with changes in the subject's capacity to perform the operant response (Edmonds \& Gallistel, 1974; Miliaressis, Rompré, Laviolette, Philippe, \& Coulombe, 1986; Stellar, Waraczynski, \& Wong, 1988; Valenstein, $1964)$, rendering the interpretation of results ambiguous.

In contrast, the curve-shift method permits changes in the rewarding efficacy of the stimulation to be dissociated from changes in the subject's capacity to perform the operant response. The experimenter determines the range of stimulation frequencies over which the rate of leverpressing varies from zero to maximum. Changes in the position of the rising portion of the rate-frequency function along the frequency axis are thought to reflect changes in the rewarding impact of the stimulation, while changes in asymptotic rate are thought to indicate alterations in the strength of the priming effect (Gallistel, 1983; Gallistel, Stellar, \& Bubis, 1974) or in the subject's capacity to perform the operant response. Validation studies have shown that the position of the rate-frequency function along the frequency axis is relatively insensitive to manipulations of the task difficulty (Edmonds \& Gallistel, 1974; Miliaressis et al., 1986; Stellar et al., 1988).

In several studies, the curve-shift method has been employed to test the hypothesis that neurons with cell bodies located in the septal complex and basal forebrain contribute to the rewarding effect of MFB stimulation. Knife cuts that damaged septal sources of MFB fibers or disconnected these nuclei from the MFB failed to produce substantial, long-lasting increases in the frequency threshold (Waraczynski, 1988). Lesions or knife cuts in the lateral preoptic area (LPO) and anterior LH (Janas \& Stellar, 1987; Stellar \& Neeley, 1982; Waraczynski, 1988) decreased the rewarding effectiveness of stimulating the caudal MFB in some subjects but were ineffective in others. More anterior lesions and knife cuts generally had no effect on the frequency threshold.

Changes in the rate of responding for three LH currents have been observed following very large radiofrequency lesions centered in the LPO (Muñoz, Keller, \& Huston, 1985); similar results were obtained following somewhat smaller but similarly centered ibotenic acid lesions (Huston, Kiefer, Buscher, \& Muñoz, 1987). Unfortunately, it is not possible to derive from these data the magnitude of the curve shift corresponding to the observed changes in rate.

The results of these studies provide partial support for the view that cells or fibers in the region of the anterior MFB play some role in the rewarding effect of stimulating more caudal portions of the bundle. The inconsistencies in the lesion data may reflect subtle variations in the relative locations of the lesion and stimulation sites. If so, in the delineation of the anterior brain areas that contribute to MFB reward, small, well-defined lesions may be more useful than large lesions. Thus, we made small electrolytic lesions in the anterior $\mathrm{LH}$ and assessed their effect on rate-frequency curves obtained at more caudal MFB self-stimulation sites in the LH and VTA.

\section{METHOD}

\section{Subjects}

Seven male, "old colony" rats of the Long-Evans strain (Charles River Breeding Farms) served as subjects. Weight at the time of surgery varied from 350 to $440 \mathrm{~g}$. The animals, individually caged, had unlimited access to food and water and were maintained on a reverse 12:12-h light:dark cycle. All behavioral testing was carried out during the dark phase of the cycle.

\section{Surgery}

Atropine sulfate was administered $20 \mathrm{~min}$ prior to anesthesia, in order to reduce mucous secretions. Surgery was performed under sodium pentobarbitol anesthesia (Somnotol, $65 \mathrm{mg} / \mathrm{kg}$ i.p.), with supplements administered as required.

Fixed electrodes were constructed from 0.25 -mm-diameter stainless steel rods, which were insulated with Formvar except at their rounded tips. Male Amphenol pins were attached to a flexible wire soldered to the electrode. Moveable electrodes were of the type designed by Miliaressis (1981). The upper portion of the moveable electrode fit snugly within a nylon tube and was secured by a set screw; the tube was attached to the skull and anchoring screws with dental acrylic. In order to lower the electrode, a calibrated driver was attached, the set screw was loosened, and the threaded feed shaft of the driver was rotated in quarter-turn increments. Each quarter turn caused the electrode to move approximately $80 \mu \mathrm{m}$.

Fixed stimulating electrodes were aimed at the $\mathrm{LH}$, using the following level-skull coordinates: $-2.8 \mathrm{~mm}$ from bregma, $1.7 \mathrm{~mm}$ lateral to the midsagittal suture, and $7.8 \mathrm{~mm}$ below the dura mater. The coordinates for the fixed lesioning electrodes aimed at the anterior LH were $-1.3 \mathrm{~mm}$ from bregma, $2.2 \mathrm{~mm}$ lateral, and $7.8 \mathrm{~mm}$ below dura. Moveable electrodes were aimed at the anterior VTA, using the coordinates $-4.8 \mathrm{~mm}$ from bregma, $1.0 \mathrm{~mm}$ lateral, and $7.2 \mathrm{~mm}$ below dura. Petroleum jelly applied to the top of the moveable electrode shaft prevented dental acrylic from binding to the electrode. A stainless steel wire wrapped around four jeweler's screws embedded in the skull served as the anode.

After the electrodes were implanted and secured to the skull with dental acrylic, the male Amphenol pins attached to the fixed electrodes and the stainless steel wire were inserted into a nine-pin, externally threaded connector and were cemented onto the head of the rat with dental acrylic. By means of an internally threaded ring, this connector was mated firmly during testing with a matching connector mounted at the end of the stimulation cable.

\section{Stabilization}

Apparatus. Several days were allowed for recovery before testing began. Subjects were initially screened for self-stimulation in wooden boxes measuring $25 \mathrm{~cm}$ wide $\times 25 \mathrm{~cm}$ deep $\times 70 \mathrm{~cm}$ high, with Plexiglas front panels and wire-mesh floors. A Lehigh Valley rodent lever was located in the center of the left wall approximately $5 \mathrm{~cm}$ from the floor. Located $5 \mathrm{~cm}$ above the lever was a yellow "jewel" light that measured $1.5 \mathrm{~cm}$ in diameter. The stimulation cable attached to the nine-pin connector on the subject's head was connected to the stimulator with a seven-channel, slip-ring commutator fixed in the center of the ceiling of the testing cage.

Depression of the lever resulted in a $0.5-\mathrm{sec}$ train of $0.1-\mathrm{msec}$, cathodal, rectangular pulses. The temporal parameters of the stimulation were controlled by hand-set integrated circuit pulse generators. The stimulation pulses were produced by dual constant-current amplifiers (Mundl, 1980) and their amplitude set by a potentiometer. Current was monitored by measuring the voltage drop across a $1-\mathrm{k} \Omega$ resistor in series with the rat. Accumulation of charge at the electrodebrain interface was minimized by a circuit that shorted the stimulator outputs through a $1-\mathrm{k} \Omega$ resistor, except during delivery of a pulse. 
Training. Subjects were initially screened for self-stimulation, using a current of $200 \mu \mathrm{A}$ and a frequency of approximately $40 \mathrm{~Hz}$. If sniffing and exploration were observed, the current was gradually increased and conventional shaping procedures were used in the effort to train the subjects to self-stimulate. The animals were tested with various current intensities, in order to determine the range of frequencies for which each subject would reliably selfstimulate. These parameters were used as a guide for later testing with the computer-operated equipment.

If a subject could not be trained to self-stimulate for stimulation delivered through the moveable electrode, the subject was briefly anesthetized with a short-acting inhalant (Metofane) and the electrode was lowered by $320 \mu \mathrm{m}$. Screening was then repeated as above.

\section{Data Collection}

Self-stimulation data were collected, using the VTA and/or the more caudal of the $\mathrm{LH}$ electrodes.

Apparatus. The computer-operated setup used to collect the data was similar to the hand-operated equipment used for screening and training. Only the aspects of the computer-operated setup that differ from the hand-operated setup will be described below.

Test chambers for the computer-operated setup consisted of Plexiglas boxes that measured $25 \times 25 \times 75 \mathrm{~cm}$, with hinged doors on the upper half of the front face and with removable floors. Lehigh Valley rodent levers were located on opposite walls of each test box, $5 \mathrm{~cm}$ from the floor and $5 \mathrm{~cm}$ from the nearest corner. A yellow jewel light measuring $1.5 \mathrm{~cm}$ in diameter was located $3 \mathrm{~cm}$ above one lever, and a red jewel light was similarly placed above the other lever (which was not used in this experiment). The test chambers were mounted in $50 \times 50 \times 90 \mathrm{~cm}$ plywood boxes insulated with $2.5 \mathrm{~cm}$ of Styrofoam. Removable front panels with Plexiglas inserts allowed the experimenter to view the subject from an adjoining room by means of a remote-controlled video camera. A single 40 -W bulb illuminated the test chamber, and an $11.5-\mathrm{cm}$ fan provided ventilation.

Temporal parameters of the stimulation for each test cage were controlled by a dedicated microprocessor with a custom-built interface. A bank of relays controlled by the parallel port of the dedicated microprocessor determined which electrode would deliver the stimulation. Stimulation current was determined by a digital-toanalog converter attached to a voltage-controlled constant-current amplifier (a modified version of Mundl's [1980] design).

Procedure. The threshold frequency was determined by holding the current constant and varying the frequency from trial to trial. At the beginning of each 30 -sec trial, the overhead light was extinguished for $0.5 \mathrm{sec}$, and five trains of priming stimulation were then delivered. Each train of priming stimulation was identical to the stimulation that would be available to the animal during the rest of the trial. Following the priming stimulation, the yellow light located above the lever was illuminated to signal that depression of the lever would trigger a train of stimulation.

The frequency for the first trial of each determination was chosen so that the subject would respond near its maximum rate. If, as expected, the animal responded during the first 30 -sec trial, the maximum response rate was then estimated by increasing the frequency delivered in subsequent trials in $0.1 \log _{10}$ unit steps until the number of responses did not exceed the number recorded on the previous trial by more than $10 \%$. If the rat did not respond on the first trial, the frequency was increased by $0.3 \log _{10}$ units on the next trial, and the search for the maximum response rate was continued as described above. A ceiling value was placed on the maximum frequency that could be delivered, so that excessively strong stimulation would never be delivered. After the maximum response rate had been estimated, the frequency was lowered to $0.1 \log _{10}$ units below the value tested on the first trial and then decreased in 0.1 $\log _{10}$ unit steps until the number of leverpresses was less than $10 \%$ of the maximum response rate recorded for that threshold determination. The frequency was then decreased a further $0.1 \log _{10}$ units for the final trial in the determination. Thus, a curve relating rate of lever-pressing to the frequency of the stimulation (rate-frequency curve) was obtained.

The session began with up to four threshold determinations (two for each electrode tested) that were used as warm-ups and were therefore not included in the data analysis. Four to six ratefrequency curves were collected per testing session at each site. Up to two testing sessions were run per day, separated by a rest period of at least $1 \mathrm{~h}$. In the case of 1 subject (B1), frequency thresholds were obtained at two different stimulation currents.

A lesion was made through the electrode aimed at the anterior LH after at least 5 days of prelesion testing. The first postlesion session was run approximately $1 \mathrm{~h}$ after the lesion. Following the collection of the postlesion rate-frequency functions, further lesions were made through the same electrode in some subjects.

Lesion Parameters. Direct current was passed with the lesioning electrode as the anode and the skull screws as the cathode. Initial lesion parameters varied, depending on the subject, from 0.2 to $1.0 \mathrm{~mA}$ for $10 \mathrm{sec}(\mathrm{B} 1, \mathrm{~B} 2: .3 \mathrm{~mA}$; B5: $.5 \mathrm{~mA} ; \mathrm{C8}, \mathrm{D} 1$, D3: $1.0 \mathrm{~mA}$; $\mathrm{C} 9: .2 \mathrm{~mA}$ ). Following collection of the postlesion data, second and third lesions were made in some subjects, using the same electrode but a higher current. Later lesion parameters varied, depending on the subject, from 1.0 to $2.0 \mathrm{~mA}$ for $10 \mathrm{sec}$ (B1, B2: $1.0 \mathrm{~mA}, 1.5 \mathrm{~mA}$; C8: $1.5 \mathrm{~mA}, 2.0 \mathrm{~mA}$; D3: $2.0 \mathrm{~mA}$; C9: $.5 \mathrm{~mA}, 1.0 \mathrm{~mA}$ ).

\section{Data Analysis}

The rate-frequency curves were fit with a broken-line function, as described by Gallistel and Freyd (1987). An example of such a function is shown in Figure 1. The broken-line function is composed of a straight line joining an upper and a lower asymptote. The four parameters of the broken-line function were chosen so that the residual sum of squares was minimized. The lower break-point was used to estimate the frequency required to initiate responding (threshold). Shifts in the frequency threshold were used to determine whether any change had occurred in the rewarding efficacy of the stimulation. The average frequency threshold was calculated for each day and plotted as a function of time before and after the lesion. The maximum rate for each rate-frequency curve was estimated from the upper asymptote of the broken-line function. The range over which the functions rose (the dynamic interval) was obtained
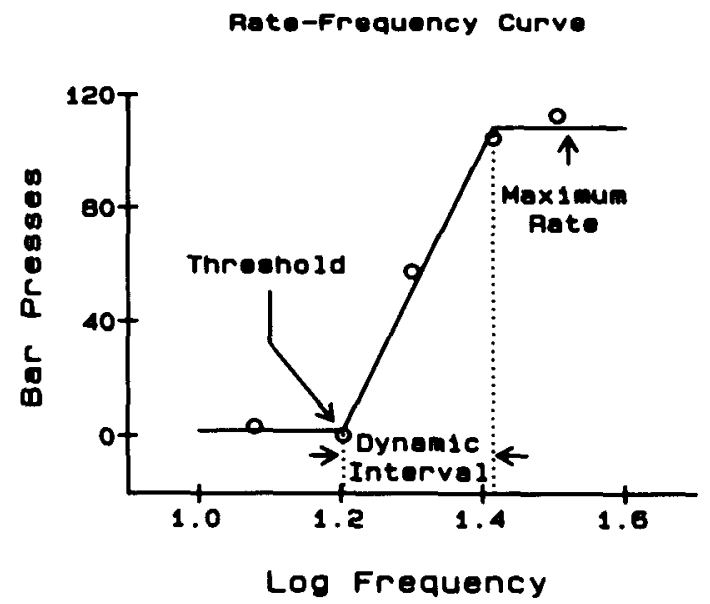

Figure 1. Example of a broken-line function fit to rate-frequency data (open circles). The function consists of a straight line joining an upper and lower asymptote. The threshold for each curve was estimated from the point of intersection of the straight line with the lower asymptote. The maximum rate for each determination was estimated from the upper asymptote and the dynamic interval from the difference between the upper and lower break-points. 


\section{Effective Lesions}

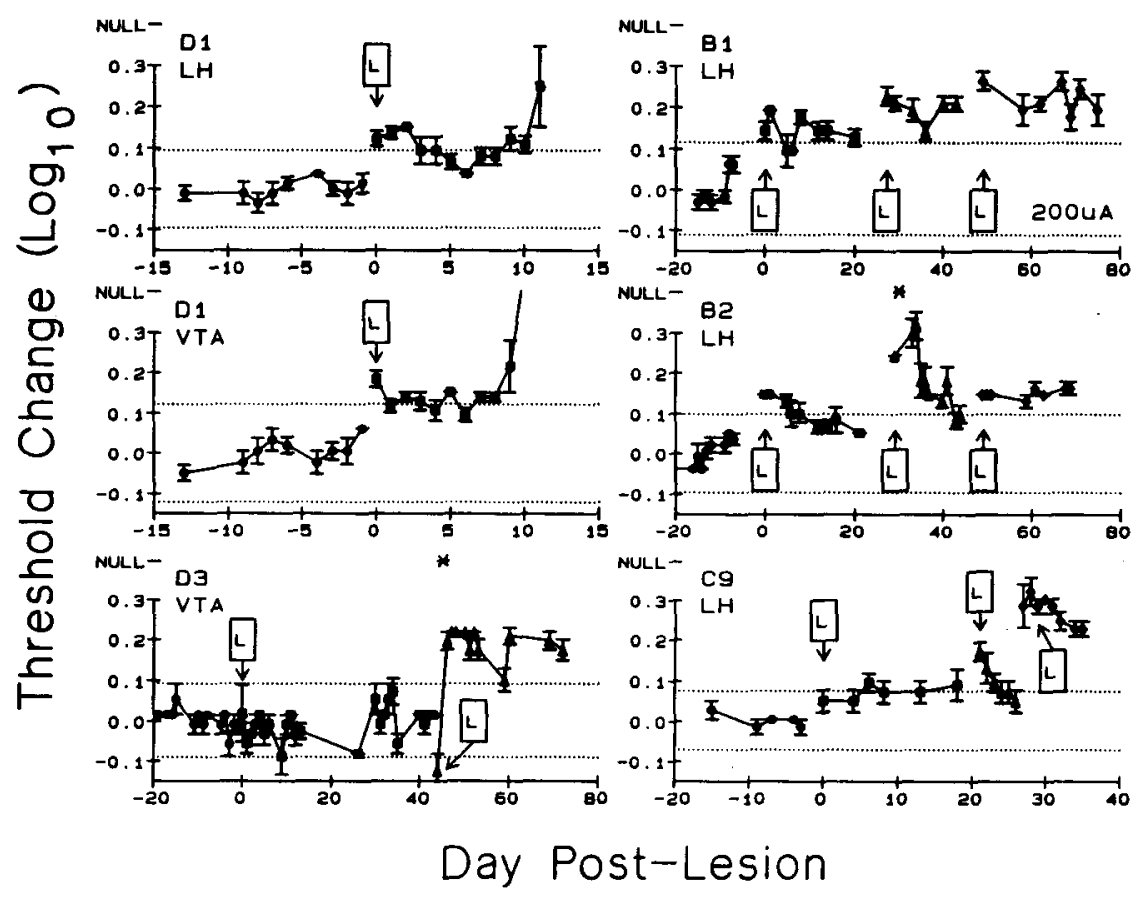

\section{Ineffective Lesions}
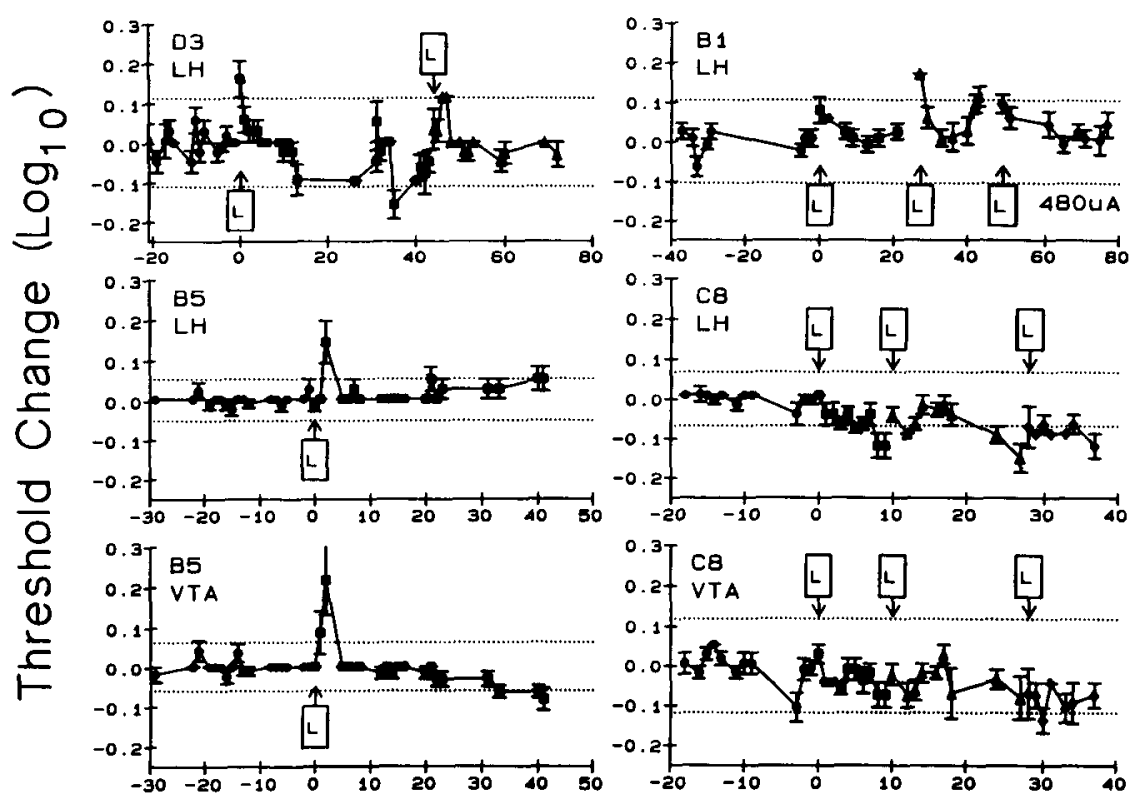

Day Post-Lesion

Figure 2. Changes in the threshold frequency for self-stimulation of the LH and VTA following lesions of the anterior LH. The days on which lesions were made are designated by the letter $L$. The top panel shows the data from subjects with effective lesions and the lower panel the data from the ineffective lesions. The horizontal, dotted lines extending across each graph indicate 2 standard deviations from the mean of the baseline data. (Baseline days have negative values along the abscissa.) Error bars around each data point represent the standard error of the mean (SEM) for that test day. Nulls are defined as test sessions in which the animal pressed (i.e., the maximum rate was greater than $20 \%$ of the baseline maximum rate) on fewer than three threshold determinations and are indicated by asterisks at the top of the graph. 
from the difference between the upper and lower break-points. If the maximum of a rate-frequency curve collected after the lesion failed to attain at least $20 \%$ of the average maximum rate for that subject during baseline testing, no broken-line function was fit. We defined the results for a given site on a given day to be "null" if brokenline functions could be fit to fewer than three rate-frequency curves.

\section{Histology}

At the completion of the experiment, the subjects were given an overdose of Somnotol (sodium pentobarbitol) and perfused intracardially with physiological saline followed by $10 \%$ formalin. The brains were removed and soaked in $10 \%$ formalin for at least 1 week. The brains were sliced in $30-\mu \mathrm{m}$-thick sections and mounted onto glass slides coated with gelatin. The sections were then stained with formol thionin. Lesions were reconstructed by locating the section with the first sign of the lesion, the largest cross-section of the lesion, and the last sign of the lesion. Landmarks located near the lesion and the electrode tips were used to identify their coordinates according to the Paxinos and Watson (1986) stereotaxic atlas.

\section{RESULTS}

Four subjects responded for stimulation of both the $\mathrm{LH}$ and the VTA, whereas 3 subjects responded only for stimulation of the LH.

\section{Effective Lesions}

Following lesions to the anterior $\mathrm{LH}$, increases in the frequency threshold were seen in the cases of two VTA sites and four LH sites (Figure 2, upper panel). Null sessions (those in which the maximum response rate was greater than $20 \%$ of the baseline maximum rate on fewer than three threshold determinations) are designated by asterisks at the top of each graph.
The increases in the frequency threshold from the baseline mean ranged from approximately $0.1 \log _{10}$ units (26\%) to $0.2 \log _{10}$ units (58\%), although transient shifts of around $0.25 \log _{10}$ units $(78 \%)$ and $0.30 \log _{10}$ units (100\%) were seen in the cases of Subjects C9 and B2, respectively. Although recovery was evident in some cases (C9, second and third lesion; B2, second lesion), the thresholds seldom returned to baseline values. In most cases, long-lasting elevations in the threshold were seen for up to 27 days of postlesion testing. The abrupt increase in threshold at the LH and VTA for Subject D1 that occurred after 10 days of postlesion testing preceded the loss of the electrode assembly. In the subjects with serial lesions through the same electrode, some of the lesions produced larger shifts in the threshold than other lesions did. For instance, Subject C9 showed a substantial shift after the third lesion, but much smaller, transient effects after the first and second lesion.

The lesion-induced increases in frequency threshold (upper panel of Figure 2) were generally not accompanied by long-lasting changes in the maximum response rate as determined from the upper asymptote of the broken-line functions. At all but one site, the depression in the maximum rate was either small (within 2 standard deviations of the baseline) or transient (lasting only a few days postlesion). Only in the case of Subject C9 was there a substantial, long-lasting decrease in the maximum rate. However, the decreases after the first and third lesions were roughly the same, despite the fact that the increase in threshold after the third lesion was much larger than it was after the first. The top row of Figure 3 shows the effect of the lesions on the maximum rate in the cases of
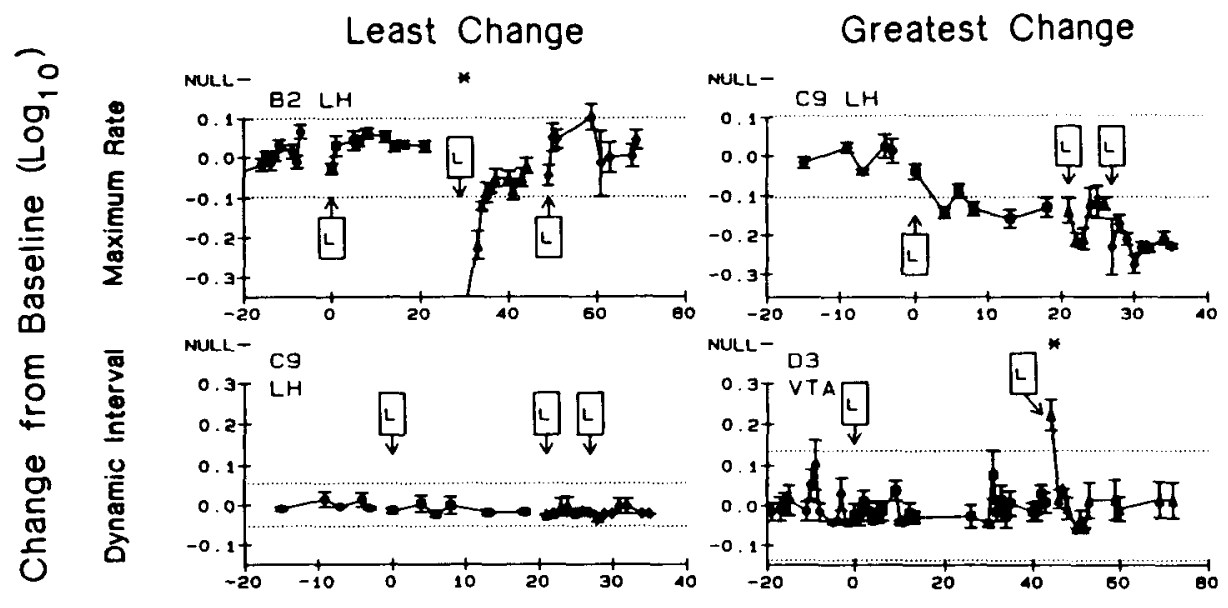

Day Post-lesion

Figure 3. Changes in the maximum rate (top row) and the dynamic interval (bottom row) following lesions to the anterior LH. Lesion days are indicated by the letter $L$. The horizontal, dotted lines extending across each graph indicate 2 standard deviations from the mean of the baseline data. Error bars around each data point represent the standard error of the mean (SEM) for that test day. Nulls are defined as test sessions in which the animal pressed (i.e., the maximum rate was greater than $20 \%$ of the baseline maximum rate) on fewer than three threshold determinations and are indicated by asterisks at the top of the graph. The least and greatest changes from baseline are shown in the left- and righthand columns, respectively. 

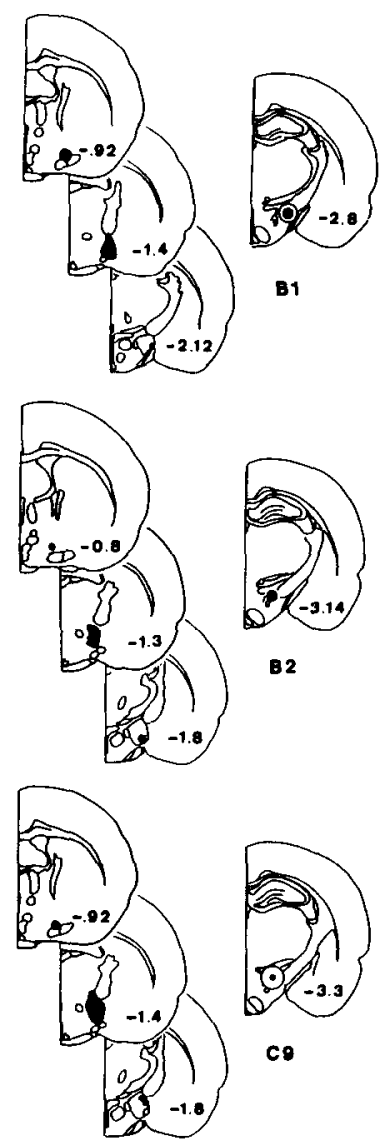

Figure 4. Lesion reconstructions (left) and LH electrode sites (right) for Subjects B1, B2, and C9 on plates from the Paxinos and Watson (1980) atlas of the rat brain. Numbers on each coronal section give the distance of the plate from bregma. Circles drawn around the electrode sites provide an estimate of the outer border of the stimulation field.

the subjects showing the least (Subject B2) and greatest (C9) change from baseline.

In all but one case, the dynamic interval of the ratefrequency curves, defined as the range of frequencies over which the broken-line functions rose, did not vary substantially from the dynamic interval of the prelesion curves. The only case where the postlesion interval deviated by more than 2 standard deviations of the baseline mean was the VTA site in Subject D3; this elevation was seen only on the 1st day after the second lesion. The lower row of Figure 3 shows the effect of the lesions on the dynamic interval in the cases of the subjects showing the least (Subject C9) and greatest (D3) change from baseline.

\section{Ineffective Lesions}

Lesions were ineffective in producing long-term increases in the frequency threshold in the cases of two VTA sites and three LH sites (Figure 2, lower panel); at an additional LH site, the lesion altered the frequency threshold corresponding to one stimulation current $(480 \mu \mathrm{A})$ but failed to alter the threshold corresponding to a lower stimulation current $(200 \mu \mathrm{A})$. (This was the only subject tested at two stimulation currents.) In the case of Subjects D3 $(\mathrm{LH})$ and $\mathrm{B} 1(\mathrm{LH}, 480 \mu \mathrm{A})$, the lesions produced transient elevations in the threshold that lasted for only a few days. It should be noted that Subject B1 was also included in the effective lesion group described above because long-term elevations in the threshold were seen at a lower current $(200 \mu \mathrm{A})$. The gradual increase in threshold seen in the case of Subject B5 (LH electrode) after 20 days of postlesion testing preceded the loss of the electrode assembly.

\section{Histology}

The first sign, largest cross-section, and last sign of the lesion, as well as the electrode locations, were reconstructed onto tracings from the Paxinos and Watson (1986) atlas of the rat brain in the coronal plane. Effective lesions are shown in Figures 4 and 5 (reconstructed lesions are shown on the left and the stimulation sites on the right), and ineffective lesions are shown in Figure 6. The parameters of the current-distance relationship obtained at the LH by Fouriezos and Wise (1984) were used to provide estimates of the radius of excitation at each stimulation site. Although these estimates pertain only to the medial/lateral axis, the stimulation fields have been drawn symmetrically in all planes for the sake of simplicity. The largest cross-section of each lesion was also reconstructed onto plates from the Nieuwenhuys, Geeraedts, and Veening (1982) atlas of the MFB (Figure 7).

Lesions were centered in the anterior $\mathrm{LH}$ between -0.8 and $-2.3 \mathrm{~mm}$ from bregma. In addition to the anterior

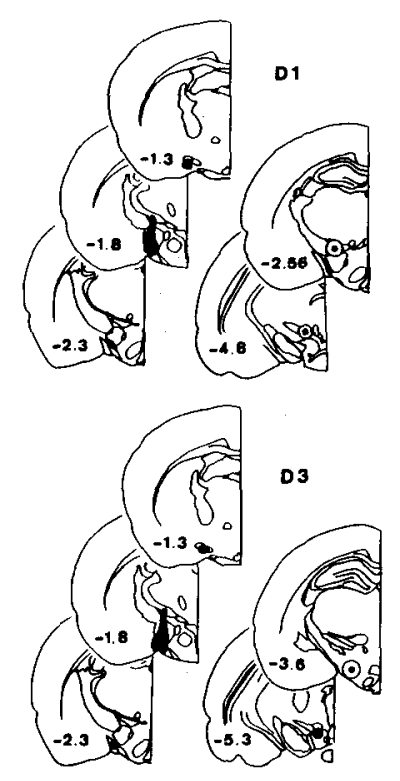

Figure 5. Lesion reconstructions (left) and LH and VTA electrode sites (right) for Subjects D1 and D3 on plates from the Paxinos and Watson (1986) atlas of the rat brain. Numbers on each coronal section give the distance of the plate from bregma. Circles drawn around the electrode sites provide an estimate of the outer border of the stimulation field. 

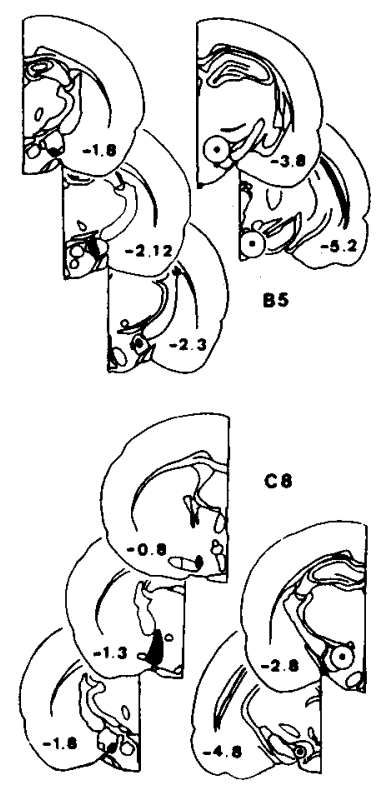

Figure 6. Lesion reconstructions (left) and LH and VTA electrode sites (right) for Subjects B5 and C8 on plates from the Paxinos and Watson (1986) atlas of the rat brain. Numbers on each coronal section give the distance of the plate from bregma. Circles drawn around the electrode sites provide an estimate of the outer border of the stimulation field.

$\mathrm{LH}$, the following areas were damaged by some, but not all, of the lesions: the lateral preoptic area (LPO); magnocellular preoptic nucleus (MCPO); horizontal limb of the diagonal band (HDB); substantia innominata (SI); nucleus of the stria medullaris (SM); medial amygdaloid nucleus, anterior (MeA); and optic tract (opt). Lesions that were ineffective in producing shifts in the frequency threshold were more medially centered in Compartment $\mathrm{C}$ of the MFB (Figure 7, bottom) while lesions that produced increases in the threshold were centered in the more lateral Compartments A, D, and E (Figure 7, top). Photomicrographs of coronal sections through an effective lesion (Subject B1, top) and an ineffective lesion (Subject C8, bottom) are shown in Figure 8. Stimulation electrodes aimed at the $\mathrm{LH}$ were located in or bordering the LH between -2.56 and $-3.8 \mathrm{~mm}$ from bregma. Stimulation electrodes aimed at the VTA were located in or bordering the VTA and were found between -4.8 and $-5.3 \mathrm{~mm}$ from bregma.

\section{DISCUSSION}

Lesions of the anterolateral portion of the MFB produced stable increases in the frequency threshold at both the LH and VTA stimulation sites. Lesions that primarily damaged more medial portions of the MFB (Compartment $C$ in the MFB atlas of Nieuwenhuys et al., 1982) failed to yield long-lasting increases in threshold at either the LH or the VTA site. In previous studies in which the curve-shift method has been employed to assess the ef- fect of anterior MFB damage on self-stimulation of more caudal MFB sites (Janas \& Stellar, 1987; Waraczynski, 1988 ), increases in frequency threshold have been found in some, but not all, subjects. The results of the present experiment suggest that the effectiveness of the lesion may depend critically on its precise location and on the choice of the stimulation current(s) tested.

\section{Contribution of Performance Variables}

In some studies of the effect of performance variables on the rate-frequency function, manipulations of the task difficulty have been found to shift the frequency threshold by as much as $0.2 \log _{10}$ units (Edmonds \& Gallistel, 1974; Fouriezos, Bielajew, \& Pagotto, 1990; Miliaressis et al., 1986). Since the magnitude of the shifts observed in this study ranged from around $0.1-0.2 \log _{10}$ units, one must consider the possibility that these shifts were due to performance deficits and not to decreases in the rewarding effect.

There are two reasons for doubting this interpretation. First, there was no marked depression in the maximum response rate after lesioning, except in the case of Subject C9. With the exception of 1 subject in the study by Fouriezos et al. (1990), performance manipulations that

EFFECTIVE LESIONS
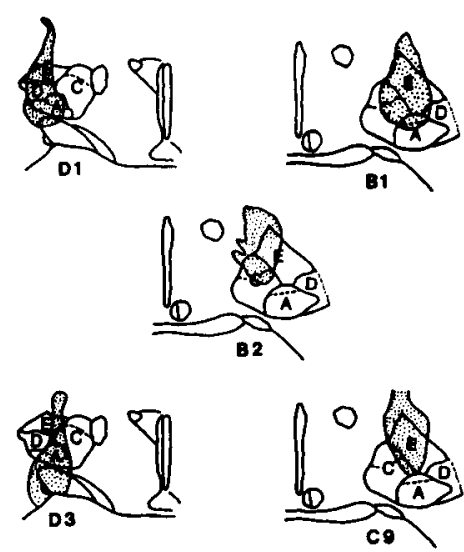

INEFFECTIVE LESIONS
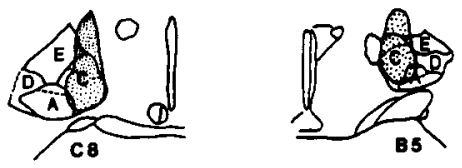

Figure 7. The location of the largest cross-section for the effective (top) and ineffective (bottom) lesions reconstructed onto plates from the Nieuwenhuys, Geeraedts, and Veening (1982) atlas of the MFB. Since the Nieuwenhuys et al. (1982) atlas of the MFB does not contain as many plates as the Paxinos and Watson (1986) atlas, there is some discrepancy between the plates chosen for the two reconstructions. 

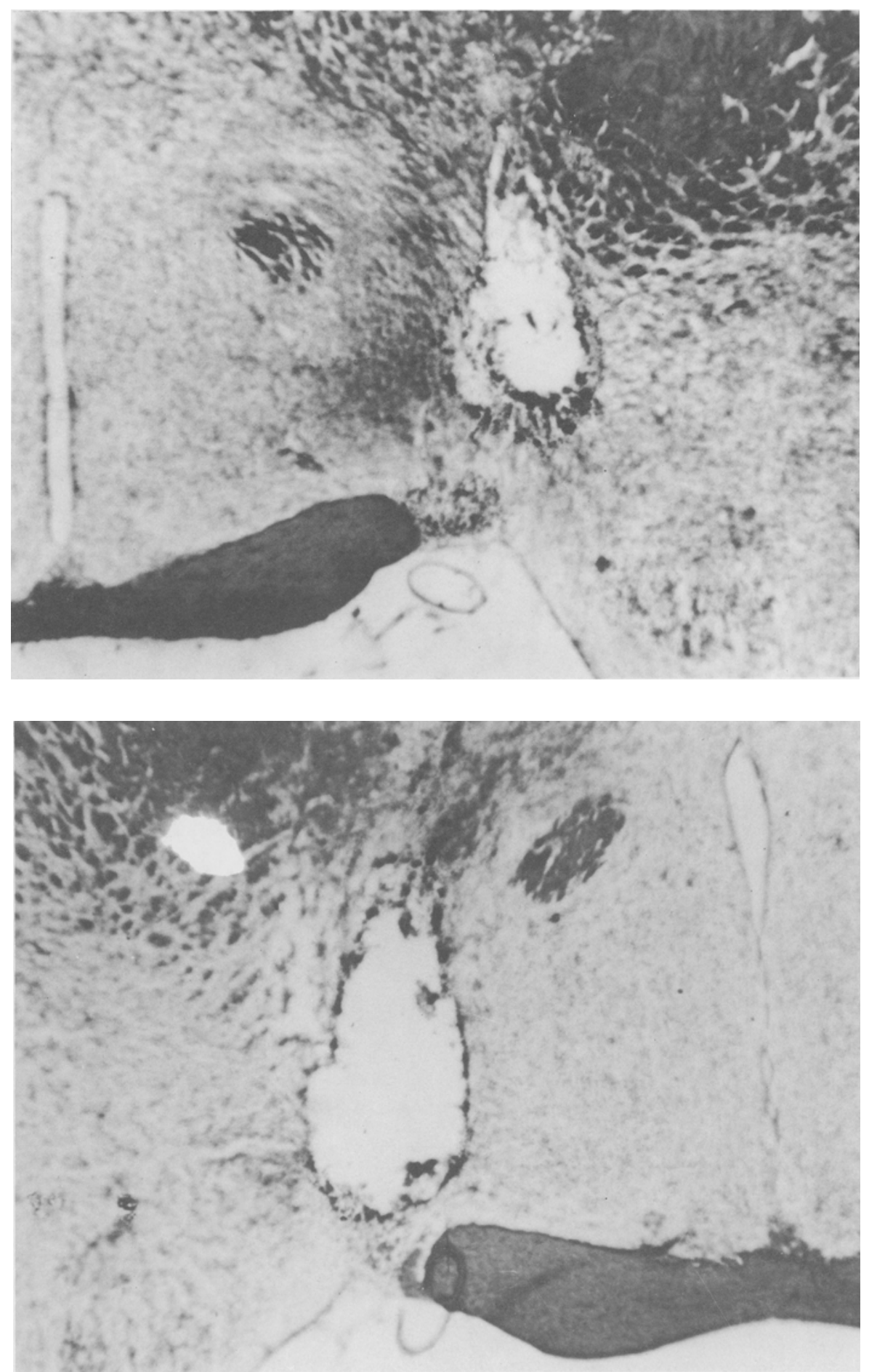

Figure 8. Photomicrographs of two coronal sections through an effective lesion (Subject B1, top) and an ineffective lesion (Subject C8, bottom). 
have increased frequency thresholds have also produced depressions in the maximum response rate. A second reason is that we found no appreciable change in the dynamic interval of the rate-frequency curves. Miliaressis et al. (1986) found that two different performance manipulations produced changes in the range over which the ratefrequency curves rose (dynamic interval), as well as in the asymptotic response rate (however, see Fouriezos et al., 1990, for an exception).

\section{Contribution of Damage at the Site of Stimulation}

Lesions of the directly stimulated region surrounding the electrode tip would increase the frequency threshold but would tell us little about the anatomy of the circuit responsible for the rewarding effect. Could such damage have contributed significantly to the observed increases in threshold? In all but one case, we suspect that this is unlikely.

The caudal border of the lesions in the present study was 2.5-3.0 mm anterior to the estimated location of the center of the VTA stimulation sites. Thus, in neither of the cases in which the lesions increased the frequency threshold at the VTA site is it likely that the effect was due to the encroachment of the lesion on the directly stimulated region surrounding the electrode tip. A similar argument can be made in the case of 3 of the subjects in which the LH thresholds increased after the lesion. In Subject B2, the caudal border of the lesion was $1.34 \mathrm{~mm}$ anterior to the estimated location of the center of the $\mathrm{LH}$ stimulation site; in Subject C9, this distance was $1.5 \mathrm{~mm}$. These values are comparable to the distances between the caudal borders of the lesions and the centers of the LH stimulation sites in the two cases in which the lesions failed to increase the frequency thresholds (Subject B5, $1.5 \mathrm{~mm}$; Subject C8, $1.0 \mathrm{~mm}$ ). Although only $0.68 \mathrm{~mm}$ separated the caudal border of the lesion from the tip of the LH stimulation electrode in Subject B1, it is unlikely that encroachment of the lesion on the directly stimulated region is responsible for the observed increase in the frequency threshold at the lower stimulation current. When the higher current was tested, the directly stimulated region should have been larger and, hence, more likely to overlap the damaged area. However, no long-lasting changes in the frequency threshold were observed at the higher current. Only in the case of the LH site in Rat D1, the center of which was separated from the caudal border of the lesion by only $0.2 \mathrm{~mm}$, is it likely that damage to the directly stimulated region contributed to the increase in the frequency threshold. Nonetheless, such an argument would not appear to hold in the case of the VTA site in this subject, a site at which a postlesion increase in threshold was also observed. Thus, the lesion in Subject D1 is of interest even if the effect at the LH stimulation site was due to local damage.

\section{Alignment of Lesions and Stimulation Sites}

In order to produce a large increase in the frequency threshold, a lesion must be aligned optimally with the reward-relevant fibers activated at the stimulation site. The small difference in the location of the effective and ineffective lesions could be interpreted to suggest that alignment is particularly crucial in the case of the lesion and stimulation sites used in the present experiment. Nonetheless, the small number of ineffective lesions and the testing of only one current per site in most cases (see below) require that these differences be interpreted with caution.

In future studies, the alignment of the lesioning and stimulating electrodes might be improved by electrophysiological means. The lesioning electrode might be positioned so as to maximize the amplitude of compound action potentials driven by the stimulation electrodes. Thus, the existence of a direct axonal linkage could be established at the time of the initial surgery. Such recordings have been obtained successfully from MFB reward sites (Kiss \& Shizgal, 1990; Shizgal, Kiss, \& Bielajew, 1982).

\section{Dependence of the Effect on \\ the Stimulation Current}

Data from Subject B1 suggest that the effects of lesions on the rate-frequency curve may be dependent on the stimulation current. At the lower current $(200 \mu \mathrm{A})$, the shifts in threshold were stable over time, whereas at the higher current $(480 \mu \mathrm{A})$, the shifts were transient, and they recovered to baseline values within a few days. If the subject had been tested only at the higher current, we might have concluded that this particular lesion was not effective in altering the rewarding value of the stimulation. Information about the location of fibers affected by the lesion can also be inferred by using multiple currents. If the lesion were to affect fibers in the outer portion of the stimulation field, increases in the frequency threshold would be expected at high currents but not at lower currents. Similarly, lesions that affect neurons located close to the tip of the stimulating electrode might result in larger increases in threshold when testing at lower currents, an effect that has been empirically demonstrated by Shizgal, Howlett, and Corbett (1979). They found that lesions of the region immediately surrounding the tip of the stimulating electrode can alter the slope of currentfrequency tradeoff functions so that the postlesion increase in the frequency threshold is larger at lower currents than at higher currents. Given these data and the results from Subject B1, it would be wise, in future lesion studies, to obtain frequency thresholds at several current intensities.

Most subjects in the present study were tested at only one current. Thus, the significance of the difference in location of the effective versus ineffective lesions should not be overemphasized. Ineffective lesions might have produced increases in the frequency threshold if subjects had been tested at other current intensities. Further studies in which small lesions are located within various MFB compartments and tests for self-stimulation are conducted at multiple currents should help determine the validity of this medial/lateral distinction. Failure to test at more than one stimulation current might also have contributed to the inconsistency of the results reported in previous studies 
of the effect of damaging the anterior MFB on selfstimulation of more caudal MFB sites (Janas \& Stellar, 1987; Stellar \& Neely, 1982; Waraczynski, 1988).

\section{The Magnitude of the Effects}

The postlesion changes in frequency threshold ranged from approximately 0.1 to $0.2 \log _{10}$ units. These changes resemble shifts that have been observed following doses of pimozide that increase the threshold without dramatically reducing the maximum rate and increasing the dynamic interval (Miliaressis et al., 1986). Under the simplest set of assumptions, the increases in frequency threshold observed following the lesions correspond to the destruction of $20 \%-37 \%$ of the directly stimulated reward neurons. This calculation is based on the assumptions that spatiotemporal integration in the substrate for the rewarding effect obeys the "counter"' model (Gallistel et al., 1981) and that damage to directly stimulated reward neurons arising, terminating, or projecting through the lesioned region was responsible for the effect of the lesions on the frequency thresholds. Of course, damage to the efferents of the directly stimulated neurons or to a pathway that enhanced transmission of the reward signal could also have contributed to the observed effects. (The reader is referred to Bielajew and Shizgal, 1986, for a more complete discussion of the anatomical interpretation of lesion-induced increases in threshold.)

\section{Optimal Lesion Size}

Given the modest postlesion increases in frequency threshold observed in the present study, one might be inclined to recommend increasing lesion size in future experiments. Although such a manipulation would be of interest, it would not be without cost, and there are reasons to doubt that it would necessarily produce larger effects.

The principal drawback to making larger lesions is the loss of anatomical precision. The difference between the locations of the effective and ineffective lesions in the present study is quite small, and would be obscured if lesion size were increased. Moreover, increasing the size of the damaged area has often failed to produce the desired result in studies of the substrate for brain stimulation reward. For example, Waraczynski (1988) found that some of her large knife cuts transecting the anterior MFB failed to decrease the rewarding effectiveness of stimulating more caudal MFB sites, even though these cuts encompassed the area damaged by smaller, effective cuts. She noted that damage to a region adjacent to the one in which some cuts were effective sometimes decreased frequency thresholds, thus raising the possibility of cancellation between antagonistic threshold-increasing and thresholddecreasing effects produced by larger lesions. Whatever the explanation, it would appear that there is no simple relationship between the extent of damage and the efficacy of anterior MFB lesions.

\section{A Role for MFB Path Neurons?}

The increases in the threshold for self-stimulation observed in the present study could have resulted from damage to cell bodies within the anterolateral MFB, terminals, or fibers of passage. The somata of the intrinsic neurons of the MFB, the so-called path neurons, are scattered throughout the lateral preoptic-hypothalamic zone and give rise to bifurcating axons with ascending and descending branches (Millhouse, 1969). These neurons have long been considered a potential component of the MFB reward system (Olds, 1962; Szabo, 1973) and should be evaluated as possible contributors to the effects observed in this experiment. In autoradiographic studies, it has been found that path neurons located in the LPO and LH send projections past the VTA (Arbuthnott, Mitchell, Tulloch, \& Wright, 1976; Hosoya \& Matsushita, 1981). Thus, the axons of path neurons directly link the LH and VTA, a characteristic of at least some of the cells responsible for self-stimulation of the MFB (Bielajew \& Shizgal, 1982; Shizgal et al., 1980).

In most studies in which MFB self-stimulation was challenged with injections of neurotoxins into the MFB, ibotenic acid has served as the lesioning agent (Lestang, Cardo, Roy, \& Velley, 1985; Nassif, Cardo, Libersat, \& Velley, 1985; Velley, 1986; Velley, Chaminade, Roy, Kempf, \& Cardo, 1983). This substance is thought to kill neurons via a receptor-mediated action in the somatodendritic region and to spare fibers of passage (Schwarcz et al., 1979). However, recent studies have shown that although ibotenic acid leaves fibers of passage intact, it may trigger a process that leads to demyelination (Coffey, Perry, Allen, Sinden, \& Rawlins, 1988; Stellar, Hall, \& Waraczynski, in press; Waraczynski \& Stellar, 1987). Thus, the behavioral effects of ibotenic acid cannot be attributed solely to the destruction of path neurons; demyelination of fibers of passage may contribute as well. Indeed, Stellar et al. (in press) observed large increases ( $\geq 0.28 \log _{10}$ units) in frequency thresholds following injection of ibotenic acid or n-methyl-d,l-aspartic acid (NMDA) in the lateral hypothalamus only when the zone of demyelination extended to the electrode tip. When the zone of demyelination was separated from the electrode tip, the frequency threshold either failed to increase or increased only modestly despite massive damage to the path neurons surrounding the electrode tip. Nonetheless, in one case in which a substantial threshold increase was observed $\left(0.16 \log _{10}\right.$ units) that could not be attributed to demyelination at the electrode tip, the zone of cell death extended into the region damaged by our effective electrolytic lesions. Thus, although these data do not support the hypothesis that path neurons in caudal regions of the $\mathrm{LH}$ contribute to the rewarding effect of MFB stimulation, they leave open the possibility that path neurons in the anterior LH may play some role.

Data from a number of studies support a role in the rewarding effect of LH and VTA stimulation for neurons arising in, terminating in, or projecting through the anterolateral portion of the $\mathrm{LH}$ and/or the caudal portion of the LPO. Indeed, Bielajew, Thrasher, and Fouriezos (1987) have found evidence that the axons of reward-related neurons directly link the LPO and the anterior $\mathrm{LH}$ and that estimates of their conduction velocity and refractory period 
overlap, but are not identical to, estimates obtained at more posterior MFB sites (Fouriezos, Walker, Rick, \& Bielajew, 1987). This could mean that self-stimulation electrodes in the LPO activate neurons common to posterior MFB sites but also activate more slowly recovering neurons that do not contribute substantially to the rewarding effect of stimulating the more posterior sites. It remains to be determined whether the reward-related fibers linking the LPO to the anterior LH extend as far as the caudal LH and the VTA. In addition, it would be interesting to explore the possibility that the slowly conducting fibers described by Bielajew et al. (1987) terminate in the region of our lesions, perhaps on somata that contribute to the effects observed here. The collision technique would provide a more direct test of both of these hypotheses. Experiments involving selective activation or destruction of cell bodies in the anterolateral LH and LPO might shed additional light on the origin of the pathway(s) responsible for the increases in frequency threshold reported here as well as the collision-like effects obtained when the LH and VTA are stimulated. If such lesions reduced the magnitude of LH-VTA collision (Shizgal, 1989; Shizgal \& Murray, 1988), and if somata in the lesioned area were shown to have conduction properties consistent with those derived from the collision data, it would be highly likely that these somata contributed to the directly stimulated stage of the substrate for selfstimulation of the MFB.

\section{REFERENCES}

Arbuthnott, G. W., Mitchell, M. J., Tulloch, I. F., \& WriGHT, A. K. (1976). Efferent pathways from lateral hypothalamic neurones. Journal of Physiology, 263, 131-132.

Bielajew, C., \& Shizgal, P. (1982). Behaviorally derived measures of conduction velocity in the substrate for rewarding medial forebrain bundle stimulation. Brain Research, 237, 107-119.

Bielajew, C., \& Shizgal, P. (1986). Evidence implicating descending fibers in self-stimulation of the medial forebrain bundle. Journal of Neuroscience, 6, 919-929.

Bielajew, C., Thrasher, A., \& Fouriezos, G. (1987). Self-stimulation sites in the lateral hypothalamic and lateral preoptic areas are functionally connected. Canadian Psychology, 28 (Abstract No. 36).

BOYD, E. S., \& GARDNER, L. C. (1967). Effect of some brain lesions on intracranial self-stimulation in the rat. American Journal of Physiology, 213, 1044-1052.

Coffey, P. J., Perry, V. H., Allen, Y., Sinten, J., \& Rawlins, J. N. P. (1988). Ibotenic acid induced demyelination in the central nervous system: A consequence of a local inflammatory response. Neuroscience Letters, 84, 178-184.

Deutsch, J. A. (1964). Behavioral measurement of the neural refractory period and its application to intracranial self-stimulation. Journal of Comparative \& Physiological Psychology, 58, 1-9.

Edmonds, D. E., Gallistel, C. R. (1974). Parametric analysis of brain stimulation reward in the rat: III. Effect of performance variables on the reward summation function. Journal of Comparative \& Physiological Psychology, 87, 876-883.

Fouriezos, G., Bielajew, C., \& Pagotto, W. (1990). Task difficulty increases thresholds of rewarding brain stimulation. Behavioural Brain Research, 37, 1-7.

Fouriezos, G., Walker, S., Rick, J., \& Bielajew, C. (1987). Refractoriness of neurons mediating intracranial self-stimulation in the anterior basal forebrain. Behavioural Brain Research, 24, 73-80.
Fouriezos, G., WiSe, R. A. (1984). Current-distance relation for rewarding brain stimulation. Behavioural Brain Research, 14, 85-89.

Gallistel, C. R. (1975). Motivation as a central organizing process: The psychophysical approach to its functional and neurophysiological analysis. In J. Cole \& T. Sonderegger (Eds.), The Nebraska Symposium on Motivation 1974, Vol. 22 (pp. 183-250). Lincoln, NE: University of Nebraska Press.

Gallistel, C. R. (1983). Self-stimulation. In J. A. Deutsch (Ed.), The physiological basis of memory (pp. 269-349). New York: Academic Press.

Gallistel, C. R., \&Reyd, G. (1987). Quantitative determination of the effects of catecholaminergic agonists and antagonists on the rewarding efficacy of brain stimulation. Pharmacology, Biochemistry \& Behavior, 26, 731-741.

Gallistel, C. R., Shizgal, P., \& Yeomans, J. S. (1981). A portrait of the substrate for self-stimulation. Psychological Review, 88, 228-273.

Gallistel, C. R., Stellar, J. R., * Bubis, E. (1974). Parametric analysis of brain stimulation reward in the rat: $I$. The transient process and the memory-containing process. Joumal of Comparative \& Physiological Psychology, 87, 848-859.

HosoyA, Y., MAtsushitA, M. (1981). Brainstem projections from the lateral hypothalamic area in the rat, as studied with autoradiography. Neuroscience Letters, 24, 111-116.

Huston, J. P., Kiefer, S., Buscher, W., \& MuÑoz, C. (1987). Lateralized functional relationship between the preoptic area and lateral hypothalamic reinforcement. Brain Research, 436, 1-8.

JaNAS, J., Stellar, J. R. (1987). Effects of knife-cut lesions of the medial forebrain bundle in self-stimulating rats. Behavioral Neuroscience, 101, 832-845.

Kiss, 1., Shizgal, P. (1990). Compound action potentials recorded in the ventral tegmental area, substantia nigra, and periaqueductal gray following rewarding stimulation of the lateral hypothalamus in the rat. Psychobiology, 18, 205-214.

Lestang, I., Cardo, B., Roy, M. T., Velley, L. (1985). Electrical self-stimulation deficits in the anterior and posterior parts of the medial forebrain bundle after ibotenic acid lesion of the middle lateral hypothalamus. Neuroscience, 15, 379-388.

LORENS, S. A. (1966). Effect of lesions in the central nervous system on lateral hypothalamic self-stimulation in the rat. Journal of Comparative \& Physiological Psychology, 62, 256-262.

MiLAARESSIS, E. (1981). A miniature, moveable electrode for brain stimulation in small animals. Brain Research Bulletin, 7, 715-718.

Miliaressis, E., Rompré, P.-P., Laviolette, P., Philippe, L., \& Coulombe, D. (1986). The curve-shift paradigm in self-stimulation. Physiology \& Behavior, 37, 85-91.

MiLlHousE, O. E. (1969). A golgi study of the descending medial forebrain bundle. Brain Research, 15, 341-363.

MundL, W. J. (1980). A constant-current stimulator. Physiology \& Behavior, 24, 991-993.

Muñoz, C., Keller, I., \&uston, J. P. (1985). Evidence for a role of the preoptic area in lateral hypothalamic self-stimulation. Brain Research, 358, 85-95.

Nassif, S., Cardo, F., Libersat, F., \& Velley, L. (1985). Comparison of deficits in electrical self-stimulation after ibotenic acid lesion of the lateral hypothalamus and the medial prefrontal cortex. Brain Research, 332, 247-257.

NieuWenhuys, R., Geeraedts, L. M. G., \& Veening, J. G. (1982). The medial forebrain bundle of the rat: I. General introduction. Joumal of Comparative Neurology, 206, 49-81.

OLDS, J. (1962). Hypothalamic substrates of reward. Physiological Reviews, 42, 554-604.

Olds, J., Milner, P. (1954). Positive reinforcement produced by electrical stimulation of septal area and other regions of rat brain. Journal of Comparative \& Physiological Psychology, 47, 419-427.

OLDs, M. E., OLDs, J. (1969). Effects of lesions in medial forebrain bundle on self-stimulation behavior. American Journal of Physiology, 217, 1253-1264.

Paxinos, G., \& WATson, C. (1986). The rat brain in stereotaxic coordinates (2nd ed.). Sydney: Academic Press. 
RoLLS, E. T. (1974). The neural basis of brain-stimulation reward. In G. A. Kerkut \& J. W. Phillis (Eds.), Progress in Neurobiology (pp. 73-153). New York: Pergamon.

ROMPRÉ, P.-P., \& SHIZGAL, P. (1986). Electrophysiological characteristics of neurons in forebrain regions implicated in self-stimulation of the medial forebrain bundle in the rat. Brain Research, 364, 338-349.

SChWarCZ, R., Hokfelt, T., FuXe, K., Jonsson, G., Goldstein, M., \& TERENIUS, L. (1979). Ibotenic acid-induced neuronal degeneration: A morphological and neurochemical study. Experimental Brain Research, 37, 199-216.

ShizgAL, P. (1989). Toward a cellular analysis of intracranial selfstimulation: Contributions of collision studies. Neuroscience \& Biobehavioral Reviews, 13, 81-90.

Shizgal, P., Bielajew, C., Corbett, D., Skelton, R., \& Yeomans, J. (1980). Behavioral methods for inferring anatomical linkage between rewarding brain stimulation sites. Journal of Comparative \& Physiological Psychology, 94, 227-237.

Shizgal, P., Howlett, S., \& CoRbetT, D. (1979). Behavioural inference of current-distance relationships in rewarding electrical stimulation of the rat hypothalamus. Paper presented at the annual meeting of the Canadian Psychological Association, Quebec City.

Shizgal, P., Kiss, I., \& Bielajew, C. (1982). Psychophysical and electrophysiological studies of the substrate for brain stimulation reward. In B. G. Hoebel \& D. Novin (Eds.), The neural basis of feeding and reward (pp. 419-429). Brunswick, ME: Haer Institute.

Shizgal, P., \& Murray, B. (1988). Modulation of collision effects by lesions: Implications for identifying neurons subserving brainstimulation reward. Society for Neuroscience Abstracts, 14, 443.6.

SHIzGal, P., \& MURRaY, B. (1989). Neuronal basis of intracranial selfstimulation. In J. M. Liebman \& S. J. Cooper (Eds.), The neuropharmacological basis of reward (pp. 107-163). New York: Oxford University Press.

Shizgal, P., SCHINDler, D., \& Rompré, P.-P. (1989). Forebrain neurons driven by rewarding stimulation of the medial forebrain bundle in the rat: Comparison of psychophysical and electrophysiological estimates of refractory periods. Brain Research, 499, 234-248.

Stellar, J. R., Hall, F. S., \& Waraczynski, M. (in press). The effects of excitotoxin lesions of the lateral hypothalamus on selfstimulation reward. Brain Research.

Stellar, J. R., \& NeEleY, S. P. (1982). Reward summation function measurements of lateral hypothalamic stimulation reward: Effects of anterior and posterior medial forebrain bundle lesions. In B. G. Hoebel \& D. Novin (Eds.), The neural basis of feeding and reward (pp. 431443). Brunswick, ME: Haer Institute.

Stellar, J. R., Waraczynski, M., \& Wong, K. (1988). The reward summation function in hypothalamic self-stimulation. In M. L. Commons, R. M. Church, J. R. Stellar, \& A. R. Wagner (Eds.), Quantitative analyses of behavior (pp. 31-58). Hillsdale, $\mathrm{NJ}$ : Erlbaum.

Szabo, I. (1973). Path neuron system of medial forebrain bundle as a possible substrate for hypothalamic self-stimulation. Physiology \& Behavior, 10, 315-328.

VALENSTEIN, E. (1964). Problems of measurement and interpretation with reinforcing brain stimulation. Psychological Review, 71, 415-437.

VELLEY, L. (1986). Effects of ibotenic acid lesion in the basal forebrain on electrical self-stimulation in the middle part of the lateral hypothalamus. Behavioural Brain Research, 20, 303-311.

Velley, L., Chaminade, C., Roy, M. T., KempF, E., \& Cardo, B. (1983). Intrinsic neurons are involved in lateral hypothalamic selfstimulation. Brain Research, 268, 79-86.

WARACZYNSKI, M. A. (1988). Basal forebrain knife cuts and medial forebrain bundle self-stimulation. Brain Research, 438, 8-22.

WARACZYNSKI, M. A., \& STELlar, J. R. (1987). Ibotenic acid lesions and lateral hypothalamic self-stimulation. Society for Neuroscience Abstracts, 13, 1324.

YEOMANS, J. (1975). Quantitative measurement of neural post-stimulation excitability with behavioral methods. Physiology \& Behavior, 15, 593-602.

YEOMANS, J. (1979). The absolute refractory periods of self-stimulation neurons. Physiology \& Behavior, 22, 911-919.

(Manuscript received March 23, 1990; revision accepted for publication November 28,1990 .) 